\title{
Associação da força muscular com fatores sociodemográficos e estilo de vida em adultos e idosos jovens no Sul do Brasil
}

\author{
The association between muscle strength and sociodemographic \\ and lifestyle factors in adults and the younger segment \\ of the older population in a city in the south of Brazil
}

Tiago Rodrigues de Lima ${ }^{1}$ Diego Augusto Santos Silva ${ }^{1}$ Douglas Francisco Kovaleski ${ }^{1}$ David Alejandro González-Chica ${ }^{2}$

${ }^{1}$ Programa de PósGraduação em Saúde Coletiva, Universidade Federal de Santa Catarina. R. Eng. Agronômico Andrei Cristian Ferreira s/n, Trindade. 88040-900 Florianópolis SC Brasil. tiagopersonaltrainer@ gmail.com

${ }^{2}$ NHMRC Centre of Research Excellence to Reduce Inequality in Heart Disease, School of Medicine, The University of Adelaide. Adelaide SA Austrália.

\begin{abstract}
Adequate muscular strength is required to perform daily activitiesand is considered a marker of overall health. The aim of this study was to identify sociodemographic and lifestyle factors associated with handgrip strength (HGS) in adults and the younger segment of the older population. A cross-sectional,population-based study was conducted with 705 individuals aged between 25 and 65 years in the city of Florianópolis, capital of the State of Santa Catarina, Brazil.HGS was assessed usinga manual hand dynamometer. Interviews were conducted with the participants to collect sociodemographic and lifestyle data.Multiple linear regressionwas performed to identify the predictors of HGS. The findings revealed that women and individuals from older age groups showed lower HGS, while being active during leisure time was associated with higher HGS.Interventions aimed at maintaining HGS levels in individuals should pay special consideration toaging and individuals who are physically inactive or insufficiently active during leisure time.
\end{abstract}

Key words Muscle strength dynamometer, Epidemiology, Cross-sectional studies, Hand strength, Public health
Resumo A força muscular é necessária para realização de atividades diárias, sendo considerada marcador de saúde global. O objetivo deste estudo foi identificar os fatores sociodemográficos e do estilo de vida correlatos à força de preensão manual (FPM) em adultos e idosos jovens. Estudo de base populacional com delineamento transversal, realizado com 705 indivíduos, de 25 a 65 anos de idade, da cidade de Florianópolis, SC, Brasil. Analisou-se a FPM por meio do instrumento de dinamometria manual. Os dados sociodemográficos e do estilo de vida foram levantados por entrevista. Utilizou-se regressão linear múltipla para a identificação das variáveis preditoras da FPM. Mulheres e individuos mais velhos foram os fatores associados a menores valores de FPM. Além disso, ser ativo no lazer foi associado a maiores valores de FPM. Intervenções objetivando a manutenção dos níveis de FPM nos indivíduos devem considerar o envelhecimento e aqueles inativos e insuficientemente ativos fisicamente no lazer.

Palavras-chave Dinamômetro de força muscular, Epidemiologia, Estudos transversais, Força da mão, Saúde pública 


\section{Introdução}

A força muscular é importante indicativo de saúde global para ambos os sexos ${ }^{1,2}$. Níveis adequados de força muscular são necessários para a independência funcional do indivíduo na realização de tarefas diárias, laborais, recreativas e desempenho físico ${ }^{3}$. Baixos níveis de força muscular foram associados à osteoporose, síndrome metabólica, infarto do miocárdio, acidente vascular encefálico e mortalidade cardiovascular em adultos de ambos os sexos ${ }^{1,4}$.

Além dos agravos citados, outros fatores foram associados a baixos níveis de força de preensão manual, como maior incidência de quedas, dependência funcional, período de recuperação hospitalar prolongado, piora da qualidade de vida, aumento nos níveis pressóricos e aumento da concentração de colesterol total ${ }^{2,5}$.

Em pessoas adultas, a força de preensão manual atinge o ápice por volta da quarta década de vida e, após este período, segue tendência gradual de decréscimo para ambos os sexos, haja vista o processo de atrofia muscular atrelado ao envelhecimento $^{6}$. Além da relação curvilínea da força de preensão manual com a idade, outros fatores sociodemográficos (sexo e nível econômico) e também do estilo de vida (diminuição do número de horas de sono/dia, tabagismo e menores níveis de atividade física) foram relatados como associados a baixos níveis de força muscular ${ }^{6-10}$.

Diversos estudos buscaram identificar a relação entre baixos níveis de força de preensão manual e variáveis sociodemográficas e do estilo de vida, entretanto, grande parte das informações são provenientes de inquéritos realizados em países de alta e média renda ${ }^{4,7,8,11,12}$ e amostras não representativas daquelas populações ${ }^{6,12,13}$.

Além das limitações existentes nos estudos publicados previamente $e^{4,7,8,11,12}$, outra situação existente na literatura é a mudança temporal dos valores de força de preensão manual. Estudo realizado no Canadá identificou diminuição de aproximadamente $10 \%$ nos níveis de força de preensão manual em adultos durante o período de 1981 a 2007-2008 ${ }^{11}$. Esse estudo é um dos poucos que investigou a tendência temporal. Assim como a redução temporal dos níveis de força de preensão manual é possível que os fatores associados à força também possam ter se modificado, haja vista mudanças no estilo de vida e na carga global de doenças observadas nas duas últimas décadas ${ }^{14}$, o que torna relevante identificar se os determinantes de determinado desfecho também estão sendo diferentes.
Diante das evidências a respeito dos efeitos para a saúde associados como consequência dos baixos níveis de força de preensão manual, torna-se relevante estudar este tema e identificar os fatores correlatos a tal condição, com o objetivo de incentivar ações de prevenção e promoção de saúde para minimizar futuros gastos decorrentes do tratamento desses danos ${ }^{15}$. O presente estudo difere dos demais ${ }^{7,9,12,16,17}$, pois se propõe a investigar concomitantemente variáveis sociodemográficas e do estilo de vida como determinantes da força de preensão manual em adultos. Outros estudos $^{7,9,12,16,17}$ investigaram de forma separada esses construtos, o que não permite afirmar que, em um modelo ajustado de análise, as associações se manteriam. Portanto, o objetivo do presente estudo foi identificar os fatores sociodemográficos e do estilo de vida associados à força de preensão manual em adultos e idosos jovens de uma cidade do sul do Brasil.

\section{Métodos}

O presente estudo transversal analítico é derivado do estudo de coorte de base populacional EpiFloripa Adultos, e investiga a terceira onda realizada em 2014/2015. O EpiFloripa Adultos tinha o objetivo de verificar a prevalência de agravos em saúde, investigar fatores de proteção e risco à saúde em adultos residentes no município de Florianópolis, Brasil. A cidade é a capital do estado de Santa Catarina, com aproximadamente 421.240 habitantes, renda mensal per capita de $\mathrm{R} \$ 1.770,20$, índice de desenvolvimento humano municipal de 0,847 , razão de dependência de 41,6\% e índice de Gini de 0,547 em $2010^{18}$.

A primeira onda do estudo ocorreu durante o período de Agosto de 2009 a Janeiro de 2010, incluindo amostra sistemática de 1.720 adultos, de 20 a 59 anos de idade, representativa de todas as regiões, condições sociais e econômicas da cidade. Informações metodológicas sobre a primeira onda do estudo podem ser acessadas na literatura ${ }^{19,20}$.

A coleta de dados do estudo "EpiFloripa Adulto 2014/2015" (terceira onda da coorte) teve seu início no mês de Agosto de 2014 e término no mês de Junho de 2015. Todos os indivíduos investigados em 2009 foram considerados elegíveis. Indivíduos incapazes de permanecer nas posições necessárias para realizar as mensurações antropométricas, incapazes de responder o questionário, amputados ou acamados foram considerados inelegíveis para o estudo. 
Os avaliadores/entrevistadores realizaram treinamento prévio para padronização de todos os testes e informações a serem coletadas. Os participantes foram contatados via telefone para perguntar sobre a intenção de participar no estudo e agendar as avaliações. Diferentemente das duas ondas anteriores do estudo (2009 e 2012) quando foram realizadas entrevistas domiciliares, em 2014/2015 os participantes se deslocaram até a Universidade Federal de Santa Catarina (UFSC) para realizar diversas avaliações clínicas (testes de flexibilidade, força de preensão manual, densitometria, ultrassom de carótida, velocidade de onda de pulso, exames de sangue e aferição de massa corporal, estatura e pressão arterial). Nos últimos dois meses de coleta de dados, entrevistadores treinados se deslocaram até o domicilio dos participantes para realizar as entrevistas e obter informações antropométricas e de pressão arterial daqueles membros da coorte que não tinham a disponibilidade de se deslocar até o local da coleta de dados.

Um total de 852 indivíduos participou da terceira onda do estudo $(49,5 \%$ da coorte original), dos quais 705 apresentavam informações completas para o presente estudo $(82,7 \%$ dos entrevistados em 2014/15). O cálculo do poder foi estimado a posteriori tendo como base este número, assim como as prevalências das distintas variáveis de exposição, do desfecho, um efeito de delineamento de 1,2 e um alfa de $5 \%$. A associação entre força de preensão manual e as variáveis sexo, idade, renda mensal per capita, horas de sono, atividade física no lazer, atividade física no deslocamento, no trabalho e no domínio doméstico apresentaram poder estatístico superior a $80 \%$. Com relação a variável hábito de fumar, se verificou poder estatístico de 6,5\% para força de preensão manual e a combinação de resultados "até 10 cigarros e 11 cigarros ou mais".

Para registro e armazenamento dos dados, utilizou-se um Tablet da marca $S A M S U N G^{\circledR}$ (Daegu, Coréia do Sul), modelo TAB 3 eliminando-se, desta forma, a etapa de digitação dos dados.

A variável dependente foi a força de preensão manual, mensurada por meio de dinamômetro manual da marca Saehan ${ }^{\circledR}$ (Seul, Coreia do Sul), com precisão de dois quilogramas e que possui validação concorrente com dinamômetro Jamar ${ }^{\circledR}$ (Lafayette, EUA) $(r=0,976)$ e confiabilidade intraexaminador $(\mathrm{r}=0,985)^{21}$. Para cada avaliado, a abertura do dinamômetro foi ajustada tendo como referência a base do dinamômetro apoiado na palma da mão e a alça para fazer a preensão à segunda articulação interfalangeana. Para a realização da mensuração, o avaliado deveria estar em pé, com o braço estendido ao longo do corpo, não sendo permitido encostar o equipamento em qualquer outro objeto ou no corpo. Após comando verbal inicial, o avaliado realizava por cinco segundos a maior força de preensão manual contra o dinamômetro e, após verificação do resultado, alternava-se a mão, permitindo-se duas tentativas. $\mathrm{O}$ resultado era anotado em quilograma força $(\mathrm{kg} / \mathrm{f})$, sendo que o melhor resultado de cada mão foi somado, a fim de se obter a força total $^{22}$. No presente estudo, a força de preensão manual foi tratada de forma contínua.

As variáveis independentes foram o sexo (masculino; feminino); idade coletada de forma contínua em anos completos; renda mensal per capita utilizada de maneira contínua.

Os comportamentos de saúde também foram analisados, como o hábito de fumar cigarros diariamente (nunca fumou, até 10 cigarros, 11 ou mais cigarros e ex-fumante); horas de sono por dia que foi analisada de forma contínua; prática de atividade física nos domínios do lazer, deslocamento, trabalho e atividades domésticas, por meio do questionário do Sistema Brasileiro de Vigilância de Fatores de Risco e Proteção para Doenças Crônicas por inquérito telefônico ${ }^{23}$. Foi considerado ativo no lazer o sujeito que relatou atividades físicas de intensidade moderada por pelo menos 30 minutos em cinco ou mais dias por semana, ou atividades de intensidade vigorosa por pelo menos 20 minutos em três ou mais dias da semana ${ }^{24}$. No domínio deslocamento, foi considerado ativo o sujeito que praticou período igual ou superior a 150 minutos semanais de caminhada ou bicicleta como forma de deslocamento $^{24}$. No domínio trabalho foi considerado muito ativo o indivíduo que relatou andar bastante a pé e carregar peso no trabalho ao menos cinco vezes na semana ${ }^{24}$. Nas atividades domésticas, se considerou ativo, aqueles que realizavam a faxina pesada de casa ao menos um dia na semana $^{24}$. Indivíduos que não atingiram esses valores ou que não realizaram essas atividades foram considerados insuficientemente ativos naquele domínio. No domínio trabalho, aqueles que relataram andar muito ou carregar peso foram considerados muito ativos.

Foi aplicada estatística descritiva e inferencial nos dados, sendo verificada a normalidade dos dados por meio de comparação da média e mediana, skewness, kurtose e gráficos. A variável renda mensal per capita precisou ser transformada devido à distribuição não simétrica, sendo que 
a correção que melhor adequou-se foi a logarítmica. Para descrição das variáveis contínuas se utilizou média e desvio padrão.

A verificação das variáveis sociodemográficas e do estilo de vida correlatas a força de preensão manual foi realizada por meio da utilização da regressão linear simples e múltipla (desfecho contínuo), sendo apresentados os resultados como coeficientes de regressão $(\beta)$, com os seus intervalos de confiança de 95\% (IC95\%).

$\mathrm{Na}$ execução dos modelos ajustados de regressão linear, se adotou a inserção das variáveis de exposição independentemente do $\mathrm{p}$ valor da análise bruta. Foram testadas interações entre as variáveis sexo e idade entre si, e, entre as variáveis sexo e idade com as demais variáveis. As modelagens foram realizadas por meio da utilização do método backward selection, e um p valor abaixo de $20 \%$ foi adotado como critério de permanência do fator nos modelos ajustados. A significância estatística para associação foi fixado em 5\%.

A estratégia utilizada para avaliar o modelo final (modelo contendo variáveis associadas ao desfecho com $p$ valor abaixo de 0,05 na análise ajustada: sexo, idade, interação sexo e idade, além da atividade física no lazer) foi a comparação de diversos parâmetros (coeficiente de determinação ajustado, coeficientes de regressão, o critério de informação de Akaike e Bayesiano, e/ou o teste de verossimilhança) com um modelo saturado (incluindo interações entre todas as variáveis independentes) e um modelo nulo (sem variáveis independentes), e estas avaliações identificaram que as variáveis inseridas no modelo final estavam ajustadas entre si e em relação ao desfecho. Os resíduos do modelo final da regressão linear múltipla foram avaliados por heterocedasticidade e normalidade. A possível multicolinearidade das variáveis preditoras do modelo foi investigada mediante o fator de inflação da variância (VIF).

Em todas as análises foi utilizado o software Stata 12.0 (Stata Corp, College Station, Texas, EUA), sendo considerado o desenho e pesos amostrais nessas análises. Para os pesos amostrais, a probabilidade de seleção por setor censitário usada em 2009 foi combinada com a probabilidade de localização em 2014/15. Estes pesos foram recalculados considerando a estrutura populacional estimada de adultos no município em 2012 (por sexo e faixa etária).

\section{Resultados}

O valor médio da idade dos participantes do estudo EpiFloripa era 45,5 $( \pm 11,6)$ anos, sendo a maioria do sexo feminino e a mediana da renda mensal per capita foi de R\$1.500,00 (Dados não apresentados em tabela).

Ao total foram obtidas informações sobre preensão manual de 705 indivíduos $(82,7 \%$ dos entrevistados em 2014/15), a maioria dos avaliados era do sexo feminino $(57,4 \%)$, valor médio da idade $45,5( \pm 11,3)$ anos e a média da renda mensal familiar per capita era de $\mathrm{R} \$ 2.380,00$ ( \pm $2.411,00)$. Estes participantes dormiam em média $7,6( \pm 1,6)$ horas por dia. Com relação aos domínios da atividade física, $50,6 \%$ da amostra foi inativa no lazer. De cada dez indivíduos, aproximadamente nove eram inativos ou insuficientemente ativos no domínio locomoção $(86,3 \%)$. Com relação à atividade física no trabalho, $42,8 \%$ dos participantes eram inativos e, a maioria $(61,1 \%)$ era inativa ou insuficientemente ativa no ambiente doméstico (Tabela 1).

Os menores valores de força de preensão manual $(\mathrm{kg} / \mathrm{f})$ foram encontrados nas mulheres, naqueles indivíduos que fumavam 11 ou mais cigarros por dia, nos inativos fisicamente no lazer, insuficientemente ativos no deslocamento e aqueles que eram ativos fisicamente no ambiente doméstico (Tabela 1).

Os fatores associados à força de preensão manual na análise bruta foram o sexo, a idade, renda mensal per capita, hábito de fumar, atividade física no domínio do lazer, atividade física no domínio do deslocamento e atividade física no domínio doméstico (Tabela 1).

A Tabela 2 apresenta os coeficientes de regressão linear da análise ajustada e o modelo final dos fatores associados à força de preensão manual. $\mathrm{Na}$ análise ajustada, o sexo foi associado à força de preensão manual e a idade foi inversamente associada à força de preensão manual, indicando que as mulheres e os mais velhos tinham menores valores de força de preensão manual. Ser ativo no lazer foi diretamente associado à força. Diferentemente da análise bruta, na análise ajustada não houve associação entre maior renda mensal per capita, os sujeitos serem ex-fumantes, insuficientemente ativos no deslocamento e, ativos no ambiente doméstico com a força de preensão manual. Após testar possíveis interações entre as variáveis, se identificou que a diminuição dos níveis de força de preensão manual em relação ao aumento dos anos de vida, é maior nos homens em comparação às mulheres (Figura 1). $\mathrm{O}$ 
Tabela 1. Distribuição da amostra, valores médios e desvio padrão da força da força preensão manual em relação as variáveis independentes e análise de regressão linear simples dos fatores associados à força de preensão manual entre participantes do Estudo Epifloripa. Florianópolis, SC, 2014-2015.

\begin{tabular}{|c|c|c|c|c|c|c|c|}
\hline \multirow[t]{2}{*}{ Variáveis } & \multirow[b]{2}{*}{$\mathbf{n}$} & \multicolumn{4}{|c|}{$\begin{array}{l}\text { Força muscular } \\
\qquad(\mathrm{kg} / \mathrm{f})\end{array}$} & \multirow{2}{*}{$\begin{array}{c}\text { Análise Bruta } \\
\text { (IC95\%) }\end{array}$} & \multirow[b]{2}{*}{$\mathbf{p}$} \\
\hline & & Amostra & \multicolumn{2}{|c|}{ Média (D.P) } & $\mathbf{B}^{\mathrm{b}}$ & & \\
\hline Total & 705 & & 64,4 & $(22,3)$ & & & \\
\hline \multicolumn{8}{|l|}{ Sexo $(\%$, IC95\%) } \\
\hline Masculino $^{*}$ & 297 & $42,6(38,0-47,3)$ & 85,5 & $(17,2)$ & & & \\
\hline Feminino & 408 & $57,4(52,7-62,0)$ & 49,0 & $(8,9)$ & $-36,23$ & $(-38,27 ;-34,18)$ & $<0,001$ \\
\hline Idade (Média, D.P) & 705 & $45,5 \quad(11,3)$ & & - & $-0,33$ & $(-0,45 ;-0,22)$ & $<0,001$ \\
\hline Renda $\dagger$ (Média, D.P) & 705 & $2.380,0(2.411,0)$ & & - & 3,06 & $(1,45 ; 4,67)$ & $<0,001$ \\
\hline Horas de Sono (Média, D.P) & 705 & $(1,6)$ & & - & $-0,25$ & $(-1,39 ; 0,88)$ & 0,659 \\
\hline \multicolumn{8}{|l|}{ Tabagismo (\%, IC95\%) } \\
\hline Nunca fumou & 379 & $56,3(50,8-61,7)$ & 62,2 & $(22,1)$ & & & \\
\hline Até 10 cigarros & 50 & $7,7(5,1-11,5)$ & 61,5 & $(24,3)$ & $-2,67$ & $(-10,76 ; 5,42)$ & 0,002 \\
\hline 11 ou mais cigarros & 54 & $9,3(6,9-12,5)$ & 61,2 & $(19,6)$ & $-0,52$ & $(-7,07 ; 6,02)$ & \\
\hline Ex-fumante & 222 & $26,6(21,9-31,9)$ & 69,2 & $(22,0)$ & 7,21 & $(2,82 ; 11,60)$ & \\
\hline \multicolumn{8}{|l|}{ AF lazer (\%, IC95\%) } \\
\hline Inativos $\S$ & 331 & $50,6(43,5-57,7)$ & 61,0 & $(20,5)$ & & & \\
\hline Insuficientemente ativos & 157 & $21,6(17,3-26,7)$ & 67,3 & $(23,7)$ & 7,14 & $(-2,71 ; 11,57)$ & 0,002 \\
\hline Ativos & 217 & $27,8(21,8-34,5)$ & 67,8 & $(23,2)$ & 7,55 & $(2,68 ; 12,41)$ & \\
\hline \multicolumn{8}{|l|}{ AF deslocamento ${ }^{\mathrm{a}}(\%, \mathrm{IC} 95 \%)$} \\
\hline Inativos $\|$ & 359 & $57,1(49,9-64,1)$ & 70,1 & $(23,1)$ & & & \\
\hline Insuficientemente ativos & 128 & $29,2(23,1-35,9)$ & 60,5 & $(20,2)$ & $-8,54$ & $(-14,30 ;-2,79)$ & 0,031 \\
\hline Ativos & 68 & $13,7(9,6-19,2)$ & 65,2 & $(23,2)$ & $-4,88$ & $(-12,15 ; 2,38)$ & \\
\hline \multicolumn{8}{|l|}{ AF no trabalho (\%, IC95\%) } \\
\hline Inativos & 153 & $42,8(35,9-50,1)$ & 67,8 & $(23,1)$ & & & \\
\hline Ativos & 133 & $27,6(22,4-33,4)$ & 66,1 & $(22,0)$ & $-0,42$ & $(-4,76 ; 3,92)$ & 0,904 \\
\hline Muito Ativos & 171 & $29,6(23,0-37,1)$ & 67,3 & $(23,0)$ & 0,41 & $(-5,32 ; 6,14)$ & \\
\hline \multicolumn{8}{|l|}{ AF doméstica } \\
\hline Inativos $^{\star *}$, & 263 & $44,8(39,0-50,8)$ & 72,1 & $(22,4)$ & & & \\
\hline Insuficientemente Ativo & 156 & $16,3(12,8-20,3)$ & 66,7 & $(25,6)$ & $-5,41$ & $(-10,88 ; 0,05)$ & $<0,001$ \\
\hline Ativos & 286 & $38,9(32,9-45,4)$ & 56,0 & $(16,7)$ & $-15,29$ & $(-19,68 ;-10,9)$ & \\
\hline
\end{tabular}

AF: Atividade física; a: variável que apresentou maior número de questões ignoradas no instrumento ( $\mathrm{n}=150)$; b: Coeficiente de regressão; IC: Intervalo de confiança; * Valores de referência em comparação ao sexo masculino na análise bruta; † Variável logaritmizada para análise de regressão; de referência em comparação a condição inativo no lazer na análise bruta; || Valores de referência em comparação a condição inativo no deslocamento na análise bruta; Valores de referência em comparação a condição inativo no trabalho na análise bruta; **Valores de referência em comparação a condição inativos na análise bruta.

modelo final para a força de preensão manual identificou um coeficiente de determinação de 0,7068, indicando que, aproximadamente, $71 \%$ da variação da força muscular foi associada às variáveis: sexo, idade, interação sexo/idade e atividade física no lazer simultaneamente. Por meio da verificação do VIF, não se identificou multicolinearidade das variáveis inseridas no modelo final $($ VIF $=1,02)($ Tabela 2$)$.

\section{Discussão}

Os principais achados deste estudo indicaram que as mulheres e indivíduos de maior faixa etária apresentaram menores valores de força de preensão manual. Também se verificou interação entre sexo e idade, indicando que o efeito negativo da idade sobre a força muscular nos homens foi maior que nas mulheres. Ainda, se verificou que ser ativo no lazer foi associado a maiores escores de força de preensão manual. 
Tabela 2. Análise de regressão linear múltipla e modelo final dos fatores associados à força de preensão manual e variáveis sociodemográficas e do estilo de vida em adultos. Florianópolis, Brasil, 2014-2015.

\begin{tabular}{|c|c|c|c|c|c|c|}
\hline \multirow{2}{*}{ Variável } & \multicolumn{3}{|c|}{ Análise ajustada $\dagger \dagger$} & \multicolumn{3}{|c|}{ Modelo final } \\
\hline & $\boldsymbol{B}^{\mathrm{a}}$ & $\%($ IC95\%) & p & $B^{\mathrm{a}}$ & $\%(\mathrm{IC} 95 \%)$ & p \\
\hline Sexo feminino & $-37,65$ & $(-40,40 ;-34,90)$ & $<0,001$ & $-53,15$ & $(-64,26 ;-42,04)$ & $<0,001$ \\
\hline Idade (anos completos)* & $-0,34$ & $(-0,45 ;-0,22)$ & $<0,001$ & $-0,51$ & $(-0,72 ;-0,30)$ & $<0,001$ \\
\hline Renda mensal (R\$) per capita $\dagger$ & 0,20 & $(-1,30 ; 1,72)$ & 0,788 & & & \\
\hline Interação sexo*idade & & & & 0,36 & $(0,15-0,58)$ & 0,001 \\
\hline Sono $\left(\right.$ horas) ${ }^{*}$ & $-0,08$ & $(-0,81 ; 0,64)$ & 0,823 & & & \\
\hline \multicolumn{7}{|l|}{ Tabagismo } \\
\hline Até 10 cigarros $\ddagger$ & $-0,54$ & $(-4,84 ; 3,76)$ & 0,844 & & & \\
\hline 11 cigarros ou mais & $-1,95$ & $(-6,30 ; 2,39)$ & & & & \\
\hline Ex-fumante & 0,39 & $(-1,81 ; 2,59)$ & & & & \\
\hline \multicolumn{7}{|l|}{ AF Lazer } \\
\hline Insuficientemente ativo $\$$ & 0,28 & $(-3,63 ; 4,19)$ & 0,011 & $-0,18$ & $(-3,97 ; 3,93)$ & 0,013 \\
\hline Ativo & 4,43 & $(1,15 ; 7,71)$ & & 4,06 & $(1,04 ; 7,07)$ & \\
\hline \multicolumn{7}{|l|}{ AF Deslocamento } \\
\hline Insuficientemente ativo\| & $-0,50$ & $(-3,38 ; 2,36)$ & 0,654 & & & \\
\hline Ativo & $-0,66$ & $(-3,93 ; 2,61)$ & & & & \\
\hline \multicolumn{7}{|l|}{ AF Trabalho } \\
\hline Ativo & 0,51 & $(-2,06 ; 3,08)$ & 0,962 & & & \\
\hline Muito Ativo & 0,01 & $(-2,92 ; 2,93)$ & & & & \\
\hline \multicolumn{7}{|l|}{ AF Doméstico } \\
\hline Insuficientemente ativo ${ }^{* *}$ & 3,21 & $(-0,16 ; 6,59)$ & 0,262 & & & \\
\hline Ativo & 1,59 & $(-1,29 ; 4,47)$ & & & & \\
\hline $\begin{array}{l}\text { AF: Atividade Física; a: Coeficiente de } \\
\text { análise de regressão; } \$ \text { Valores de refer } \\
\text { condição inativo no lazer; \|Valores de } \\
\text { comparação a condição inativo no tra } \\
\text { Todas as variáveis foram introduzidas } \\
\text { foram retiradas do modelo ajustado. }\end{array}$ & $\begin{array}{l}\text { são; IC- } \\
\text { em comp } \\
\text { ncia em } \\
* * \text { Valore } \\
\text { delo aju } \\
\text { elo final }\end{array}$ & $\begin{array}{l}\text { rvalo de confiança; } \\
\text { cão a condição não } \\
\text { paração a condição } \\
\text { referência em com } \\
\text { o independenteme } \\
\text { nado pelas variávei }\end{array}$ & $\begin{array}{l}\text { ariável cor } \\
\text { nar; } \S \text {,Valc } \\
\text { ativo no d } \\
\text { ação a cor } \\
\text { do p valor } \\
\text { xo, idade, }\end{array}$ & $\begin{array}{l}\text { ínua; } † \\
\text { es de ref } \\
\text { locamen } \\
\text { dição ina } \\
\text { ha anális } \\
\text { teração }\end{array}$ & $\begin{array}{l}\text { ariável logaritmizada } \\
\text { rência em comparaçą } \\
\text { o; } 9 \text { Valores de referê } \\
\text { ivos no domínio don } \\
\text { bruta. Variáveis com }\end{array}$ & $\begin{array}{l}\text { para } \\
\text { lo a } \\
\text { ncia em } \\
\text { héstico; } \dagger \dagger \\
p \geq 0,20 \\
\text { le física }\end{array}$ \\
\hline
\end{tabular}

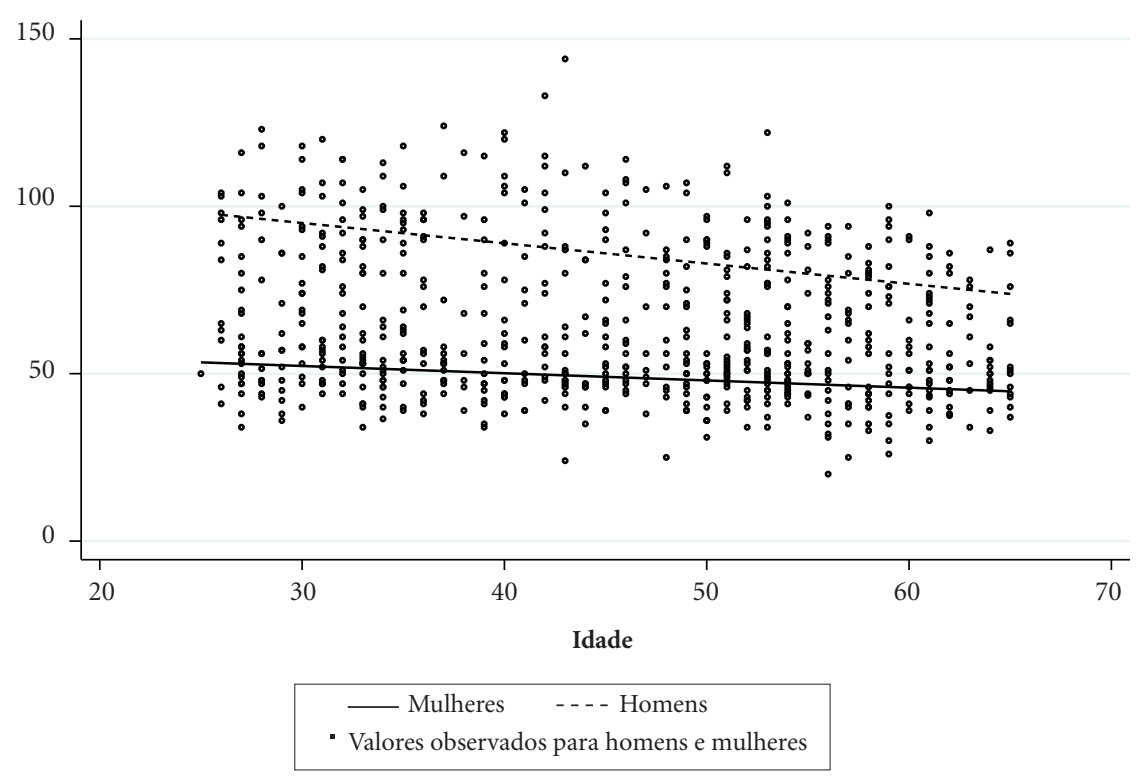

Figura 1. Relação força de preensão manual e idade, de acordo com o sexo. 
Em relação aos menores escores de força das mulheres em comparação aos homens verificados na presente pesquisa, diversos estudos identificaram resultados semelhantes ${ }^{11,12,16,25}$. A justificativa dos menores níveis de força de preensão manual por parte das mulheres seria consequência da menor quantidade de massa muscular quando comparadas aos homens ${ }^{25}$. Diversos fatores hormonais favorecem essas diferenças, tais como menor concentração plasmática dos hormônios anabólicos testosterona nas mulheres, fator de crescimento semelhante à insulina tipo 1 (IGF-1) e o hormônio do crescimento (GH ${ }^{26}$. Outro aspecto que agrava ainda mais as evidências biológicas na comparação entre homens e mulheres é que, no domínio lazer, as mulheres praticam atividade física em menor intensidade do que os homens, o que incide diretamente em menores níveis de força de preensão manual ${ }^{12,27}$. No presente estudo, a prevalência de inatividade física no lazer nas mulheres foi de $50,4 \%$ e nos homens $44,2 \%$ (dados não apresentados em Tabelas/Figuras).

O aumento dos anos de vida esteve inversamente associado à força de preensão manual. Esse achado corrobora com resultados verificados em outras pesquisas ${ }^{11,12,16,25}$. Possíveis justificativas dos menores níveis de força de preensão manual em relação à idade mais avançada seria o enfraquecimento da musculatura esquelética concomitante ao acréscimo dos anos de vida e a diminuição da qualidade e quantidade de massa muscular em decorrência da deterioração das fibras musculares ${ }^{6}$. Além disso, indivíduos adultos de maior faixa etária são mais sedentários no lazer em comparação aos mais novos ${ }^{7}$. Na presente pesquisa, foram verificadas maiores prevalências de indivíduos insuficientemente ativos no lazer nos participantes de maior faixa etária (dados não apresentados em Tabelas/Figuras).

Apesar dos homens apresentarem maiores níveis absolutos e relativos de força de preensão manual em comparação às mulheres ${ }^{4,6}$, a presente investigação encontrou que a diminuição dos níveis de força de preensão manual foi mais acentuada nos homens em comparação às mulheres à medida que se avançava a idade. $\mathrm{O}$ envelhecimento acarreta em alterações negativas no sistema neuromuscular, como capacidade de ativação de unidades motoras e fibras músculo esqueléticas, e esta condição, aparentemente, afeta em maior magnitude os homens, haja vista a maior quantidade de massa muscular em comparação às mulheres ${ }^{6}$. Além disso, o decréscimo dos níveis de produção do hormônio testosterona nos homens contribui positivamente para o aumento da concentração de massa gorda e diminuição de massa magra, o que poderia justificar a maior diminuição dos níveis de força em função da idade nos homens em comparação as mulheres ${ }^{6}$.

Foi verificado que ser ativo fisicamente no domínio lazer se associou a maiores níveis de força de preensão manual. Estes resultados concordam com os achados de outras pesquisas ${ }^{7,12}$. A prática de atividade física incide na realização de movimentos corporais e geram cargas mecânicas. Esse fato estimula o sistema músculo esquelético, levando ao aumento de massa muscular e desenvolvimento de maiores níveis de força de preensão manual ${ }^{27}$. Assim sendo, a realização de atividade física de maneira sistemática contribui positivamente para maiores níveis de força de preensão manual ${ }^{27}$.

Não se verificou relação entre força de preensão manual e as variáveis renda mensal per capita, horas de sono/dia, tabagismo. Contrariando os achados de outra pesquisa ${ }^{9}$, menor renda mensal per capita não se associou com baixos níveis de força de preensão manual. Uma possível justificativa para os achados do presente estudo seria o fato de que os indivíduos com menor renda mensal per capita estariam inseridos em piores empregos, que por sua vez, exigem maior esforço físico e conseguinte desenvolvimento de força. Os achados em relação a não associação entre força de preensão manual e horas de sono/dia poderiam ser justificados porque não se investigou a frequência semanal de vigília, fator diretamente associado a escores de força ${ }^{28}$. Apesar de ter sido identificada relação direta entre hábito de fumar e diminuição dos níveis de força em outra pesquisa $^{8}$, no presente estudo não se verificou essa associação, em que a possível justificativa para este fato, seria o baixo poder disponível para testar as associações entre estas variáveis.

Não se verificou associação entre força de preensão manual e atividade física nos domínios deslocamento, trabalho e doméstico. A utilização de instrumentos que mensuram a intensidade de determinada atividade física de maneira direta, a exemplo da utilização de acelerômetros, poderia fornecer informações específicas em relação ao deslocamento destes indivíduos, reduzindo desta forma possível viés de resposta por parte dos avaliados, sendo esta possível justificativa para não associação da atividade física e força de preensão neste domínio. Não foi verificada associação entre força de preensão manual e atividade física no domínio trabalho e ambiente doméstico, em que possível justificativa para estes fatos seriam em 
decorrência da não estratificação da amostra em relação ao número de vezes (volume) e a duração dos movimentos (intensidade) que o avaliado realizava durante o período que estava no trabalho ou durante as tarefas domésticas, fatores diretamente associados a níveis de força ${ }^{27}$. O instrumento empregado para avaliar a atividade física em tais domínios não permitia tal averiguação.

Pode-se considerar como limitação deste estudo o delineamento transversal, que impede o estabelecimento de causalidade e temporalidade entre níveis de força muscular e as demais variáveis investigadas. A causalidade reversa também não pode ser descartada, frequente na interpretação de estudos transversais ${ }^{29}$. Ademais, pode-se citar o baixo poder disponível para testar associações entre níveis de força de preensão manual com al- gumas variáveis independentes. Por fim, a não utilização de instrumentos diretos de mensuração de atividade física é tido como limitação da pesquisa.

A presente pesquisa apresenta contribuição importante para a área da saúde, pois identificou grupos suscetíveis a apresentar menores níveis de força muscular, que é indicador de saúde global. Ainda, a observação em um mesmo modelo de análise de fatores sociodemográficos e do estilo de vida pode servir de parâmetro comparativo para próximas investigações com esta população.

Pode-se concluir que mulheres, indivíduos mais velhos e aumento dos anos de vida nos homens, foram os fatores associados a menores valores de força de preensão manual. Ademais, ser ativo no domínio lazer foi associado a maiores escores de força de preensão manual.

\section{Colaboradores}

TR Lima participou da elaboração, análise, interpretação dos resultados e escrita do manuscrito. DF Kovaleski auxiliou a escrita e revisão do manuscrito. DAS Silva e DA González-Chica auxiliaram na elaboração, análise estatística, discussão e revisão do manuscrito. 


\section{Referências}

1. Leong DP, Teo KK, Rangarajan S, Lopez-Jaramillo P, Avezum A, Orlandini A, Seron P, Ahmed SH, Rosengren A, Kelishadi R, Rahman O, Swaminathan S, Iqbal R, Gupta R, Lear SA, Oguz A, Yusoff K, Zatonska K, Chifamba J, Igumbor E, Mohan V, Anjana RM, Gu H, Li W, Yusuf S; Prospective Urban Rural Epidemiology (PURE) Study investigators. Prognostic value of grip strength: findings from the Prospective Urban Rural Epidemiology (PURE) study. Lancet 2015; 386(9990):266-273.

2. Norman K, Stobäus N, Gonzalez MC, Schulzke JD, Pirlich M. Hand grip strength: outcome predictor and marker of nutritional status. Clin Nutr 2011; 30(2):135-142.

3. Forrest KY, Bunker CH, Sheu Y, Wheeler VW, Patrick AL, Zmuda JM. Patterns and correlates of grip strength change with age in Afro-Caribbean men. Age Ageing 2012; 41(3):326-332.

4. Dodds RM, Syddall HE, Cooper R, Benzeval M, Deary IJ, Dennison EM, Der G, Gale CR, Inskip HM1, Jagger C, Kirkwood TB, Lawlor DA, Robinson SM, Starr JM, Steptoe A, Tilling K, Kuh D, Cooper C, Sayer AA. Grip strength across the life course: normative data from twelve British studies. PloS One 2014; 9(12):e113637.

5. Lawman HG, Troiano RP, Perna FM, Wang C-Y, Fryar CD, Ogden CL. Associations of Relative Handgrip Strength and Cardiovascular Disease Biomarkers in US Adults, 2011-2012. Am J Prev Med 2015; 50(6):677-683.

6. Hossain MG, Zyroul R, Pereira B, Kamarul T. Multiple regression analysis of factors influencing dominant hand grip strength in an adult Malaysian population. $J$ Hand Surg Eur Vol 2012; 37(1):65-70.

7. Hansen AW, Beyer N, Flensborg-Madsen T, Grønbæk M, Helge JW. Muscle strength and physical activity are associated with self-rated health in an adult Danish population. Prev Med 2013; 57(6):792-798.

8. Kok MO, Hoekstra T, Twisk JW. The longitudinal relation between smoking and muscle strength in healthy adults. Eur Addict Res 2012; 18(2):70-75.

9. Hairi FM, Mackenbach JP, Andersen-Ranberg K, Avendano M. Does socio-economic status predict grip strength in older Europeans? Results from the SHARE study in non-institutionalised men and women aged 50+. J Epidemiol Community Health 2010; 64(9):829837.

10. Fex A, Barbat-Artigas S, Dupontgand S, Filion ME, Karelis AD, Aubertin-Leheudre M. Relationship between long sleep duration and functional capacities in postmenopausal women. J Clin Sleep Med 2012; 8(3):309-313.

11. Shields M, Tremblay MS, Laviolette M, Craig CL, Janssen I, Gorber SC. Fitness of Canadian adults: Results from the 2007-2009 Canadian health measures survey. Public Health Rep 2010; 21(1):21-15.

12. Aadahl M, Beyer N, Linneberg A, Thuesen BH, Jørgensen T. Grip strength and lower limb extension power in 19-72-year-old Danish men and women: the Health 2006 study. BMJ open 2011; 1(2):e000192.
13. Michelin E, Corrente JE, Burini RC. Fatores associados aos componentes de aptidão e nível de atividade física de usuários da Estratégia de Saúde da Família, Município de Botucatu, Estado de São Paulo, Brasil, 2006 a 2007. Epidemiol Serv Saúde 2011; 20(4):471-480.

14. GBD 2013 Risk Factors Collaborators, Forouzanfar MH, Alexander L, Anderson HR, Bachman VF, Biryukov S, Brauer M. Global, regional, and national comparative risk assessment of 79 behavioural, environmental and occupational, and metabolic risks or clusters of risks in 188 countries, 1990-2013: a systematic analysis for the Global Burden of Disease Study 2013. Lancet 2015; 386(10010):2287-2323.

15. Silva DAS, Andrade Gonçalves EC, Grigollo LR, Petroski EL. Fatores associados aos baixos níveis de força lombar em adolescentes do Sul do Brasil. Rev Paul Pediatr 2014; 32(4):360-366.

16. Massy-Westropp NM, Gill TK, Taylor AW, Bohannon RW, Hill CL. Hand Grip Strength: age and gender stratified normative data in a population-based study. $B M C$ Res Notes 2011; 4(1):127.

17. Puh U. Age-related and sex-related differences in hand and pinch grip strength in adults. Int $J$ Rehabil Res 2010; 33(1):4-11.

18. Programa das Nações Unidas para o Desenvolvimento (PNUD). Atlas do Desenvolvimento Humano, 2013. [acessado 2015 Jan 15]. Disponível em: http://www. atlasbrasil.org.br

19. Boing AC, Peres KG, Boing AF, Hallal PC, Silva NN, Peres MA. EpiFloripa Health Survey: the methodological and operational aspects behind the scenes. Rev Bras Epidemiol 2014; 17(1):147-162.

20. Peres MA, Peres KG, Boing AF, Bastos JL, Silva DA, González-Chica DA. Oral health in the EpiFloripa: a prospective study of adult health in Southern Brazil. Rev Bras Epidemiol 2014; 17(2):571-575.

21. Reis MM, Arantes PMM. Medida de força de preensão manual-validade e confiabilidade do dinamômetro Saehan. Fisioter Pesqui 2011; 18(2):176-181.

22. The Canadian Physical Activity, Fitness and Lifestyle Approach (CPAFLA). Health and Fitness Program's Health-Related Appraisal and Counselling Strategy. Ottawa: Canadian Society for Exercise Physiology (CSEP); 2003.

23. Moura EC, Morais NOL, Malta DC, Moura L, Silva NN, Bernal R, Rafael MC, Carlos AG. Vigilância de fatores de risco para doenças crônicas por inquérito telefônico nas capitais dos 26 estados brasileiros e no Distrito Federal (2006). Rev Bras Epidemiol 2008; 11(1):20-37.

24. Florindo AA, Hallal PC, Moura EC, Malta DC. Prática de atividades físicas e fatores associados em adultos, Brasil, 2006. Rev Saúde Públ 2009; 43(2):65-73.

25. Moy FM, Darus A, Hairi NN. Predictors of Handgrip Strength Among Adults of a Rural Community in Malaysia. Asia Pac J Public Health 2013; 27(2):176-184.

26. Montalcini T, Migliaccio V, Ferro Y, Gazzaruso C, Pujia A. Androgens for postmenopausal women's health? Endocrine 2012; 42(3):514-520. 
27. Chahal J, Lee R, Luo J. Loading dose of physical activity is related to muscle strength and bone density in middle-aged women. Bone 2014; 67:41-45.

28. Taheri M, Arabameri E. The effect of sleep deprivation on choice reaction time and anaerobic power of college student athletes. Asian J Sports Med 2012; 3(1):15.

29. Silva MC, Fassa AG, Valle NCJ. Chronic low back pain in a Southern Brazilian adult population: prevalence and associated factors. Cad Saude Publica 2004; 20(2):377-385.

Artigo apresentado em 21/06/2016

Aprovado em 11/11/2016

Versão final apresentada em 13/11/2016 